\title{
The Rise and Fall of NGAL in Acute Kidney Injury
}

\author{
Johan Mårtensson ${ }^{\mathrm{a}, \mathrm{d}}$ Rinaldo Bellomo ${ }^{\mathrm{a}-\mathrm{c}}$ \\ ${ }^{a}$ Department of Intensive Care, Austin Hospital, and ${ }^{\mathrm{b}}$ Australian and New Zealand Intensive Care Research Centre, \\ and ${ }^{C}$ Department of Epidemiology and Preventive Medicine, Monash University, Melbourne, Vic., Australia; \\ dSection of Anaesthesia and Intensive Care Medicine, Department of Physiology and Pharmacology, \\ Karolinska Institutet, Stockholm, Sweden
}

\section{Key Words}

Neutrophil gelatinase-associated lipocalin · Acute kidney injury $\cdot$ Intensive care $\cdot$ Inflammation

\begin{abstract}
For many years, neutrophil gelatinase-associated lipocalin (NGAL) has been considered the most promising biomarker of acute kidney injury (AKI). Commercial assays and point-ofcare instruments, now available in many hospitals, allow rapid NGAL measurements intended to guide the clinician in the management of patients with or at risk of AKI. However, these assays likely measure a mixture of different NGAL forms originating from different tissues. Systemic inflammation, commonly seen in critically ill patients, and several comorbidities contribute to the release of NGAL from haematopoietic and non-haematopoietic cells. The unpredictable release and complex nature of the molecule and the inability to specifically measure NGAL released by tubular cells have hampered its use a specific marker of AKI in heterogeneous critically ill populations. In this review, we describe the nature and cellular sources of NGAL, its biological role and diagnostic ability in AKI and the increasing concerns surrounding its diagnostic and clinical value.
\end{abstract}

(c) 2014 S. Karger AG, Basel

\section{KARGER}

E-Mail karger@karger.com

www.karger.com/bpu

\section{Introduction}

The predictive and diagnostic value of neutrophil gelatinase-associated lipocalin (NGAL) in acute kidney injury (AKI) has been assessed for more than a decade [1]. Such assessment was triggered by genomic and subsequent proteomic analyses showing that the NGAL protein was highly induced in animal kidneys and released in the urine following ischaemic or nephrotoxic insults [1, 2]. After the first promising validation study in humans [3], NGAL was for several years considered the 'troponin' of the kidney [4]. However, unlike myocardial infarction, $\mathrm{AKI}$ is rarely triggered by ischaemia. Instead, the underlying pathophysiology is characterized by a complex interaction between predisposing chronic illnesses, haemodynamic disturbances, nephrotoxic insults and inflammatory responses leading to tubular cell injury and eventually a fall in glomerular filtration rate [5]. Adding to this complexity is the fact that the NGAL molecule is produced by a number of tissues in different molecular forms $[6,7]$. Indeed, the inability to distinguish the specific molecular forms produced by the kidney from other forms released by non-renal tissues has hampered its use as reliable biomarker of AKI in the critically ill patient. 
This review summarizes the current knowledge about NGAL, its cellular origin and possible role in inflammation and organ damage with focus on AKI and highlight growing concerns surrounding the diagnostic value and clinical utility of NGAL.

\section{The Nature and Cellular Sources of NGAL in Humans}

NGAL, also known as human neutrophil lipocalin or lipocalin 2, was purified from the secondary granules of human neutrophils for the first time in the early 1990s by two independent Scandinavian groups $[8,9]$. Western blot analysis revealed that the NGAL molecule exists in three different molecular forms in blood and urine; as a $25-\mathrm{kDa}$ monomer, as a $45-\mathrm{kDa}$ disulphide-linked homodimer and as a $135-\mathrm{kDa}$ heterodimer, covalently conjugated with gelatinase [matrix metalloproteinase (MMP)-9] [8, 9].

NGAL is synthesized in the bone marrow during myelopoiesis from where it is directed to and stored in the neutrophil granules [10]. In addition, Cowland and Borregaard [6] demonstrated that NGAL mRNA is expressed in several non-haematopoietic tissues, such as colon, trachea, lung and kidney epithelium. Interleukin- $1 \beta$, an inflammatory mediator, stimulates NGAL synthesis in a number of these cell lines in vitro [7, 11]. Besides, elevated plasma levels observed during conditions such as acute peritonitis, acute exacerbations of obstructive pulmonary diseases and acute bacterial infections support its role in tissue inflammation [12-14].

The nature of the NGAL molecule is complex and seems to be related to its cellular origin. Results from in vitro experiments indicate that activated neutrophils mainly release homodimeric NGAL and to a lesser extent the monomeric form [7]. In contrast, stressed kidney epithelial cells predominantly secrete monomeric NGAL apparently unable to form dimers. This is supported by elevated urinary homodimeric levels seen in patients with urinary tract infections and a relative abundance of monomeric NGAL in AKI patients $[7,15]$. Although specific for tubular cells, the heterodimeric form seems to exist in very low concentrations, even during AKI [7].

\section{NGAL-Mediated Iron Traffic}

Several biological functions for NGAL have been suggested. By its ability to bind siderophores (small ironbinding molecules), NGAL is involved in the iron trans- port to and from cells [16]. Iron is vital for bacterial proliferation and is acquired from the host by the release of siderophores. Hence, NGAL acts as a bacteriostatic agent by sequestering iron $[17,18]$. While neutrophils provide the organism with a mobile source of NGAL, the production by epithelial cells may be important for the local defence against infections.

Excess extracellular iron may cause organ injury owing to its ability to catalyse the conversion of hydrogen peroxide to free oxygen radicals. Induced NGAL synthesis during cellular stress/inflammation may reduce extracellular iron-induced injury. Iron is also vital for the proliferation and differentiation of human cells. Yang et al. [19] found that NGAL promoted iron-dependent differentiation of mesenchymal progenitors into complete nephrons during the development of the kidneys. The renoprotective role of NGAL is supported by animal models of ischaemia-reperfusion-induced AKI, where intravenously administered NGAL was rapidly taken up by proximal tubular cells and reduced tubular damage and apoptosis as well as increased cell proliferation $[20,21]$.

\section{NGAL in Acute Kidney Injury}

During the last decade, focus has shifted and NGAL has moved from being a marker of undifferentiated systemic inflammation to become a possible marker for early detection of AKI. This was triggered by genomic analyses showing that NGAL was one of the most rapidly upregulated genes after ischaemic AKI in animals $[1,22]$. Proteomic analyses verified that the NGAL protein was induced in the kidneys following ischaemic and nephrotoxic AKI and that urinary concentration increased several-fold early on (within hours) after the insult $[1,2]$. Subsequent, studies on critically ill patients have consistently shown an association with levels in plasma or urine and severity of established AKI [23-27].

The first study evaluating NGAL as a predictor of evolving AKI was conducted on children undergoing cardiac surgery. Urinary NGAL rose almost 100 -fold and serum NGAL 20-fold up to $48 \mathrm{~h}$ before AKI was detected by creatinine. The urinary NGAL level was an almost perfect AKI predictor with an area under the receiver operating characteristics curve (ROC area) of 0.998 [3]. Since obtaining these encouraging results, the predictive performance has been tested in various clinical settings. Particularly in adult patients the results have been rather disappointing with ROC areas for urinary NGAL to predict moderately severe AKI (RIFLE R and/or AKIN stage 1) 
Fig. 1. Studies in mixed ICU and cardiac surgery patients investigating the value (ROC area) of urinary NGAL to predict AKI defined by RIFLE $\mathrm{R}$ and/or AKIN stage 1.

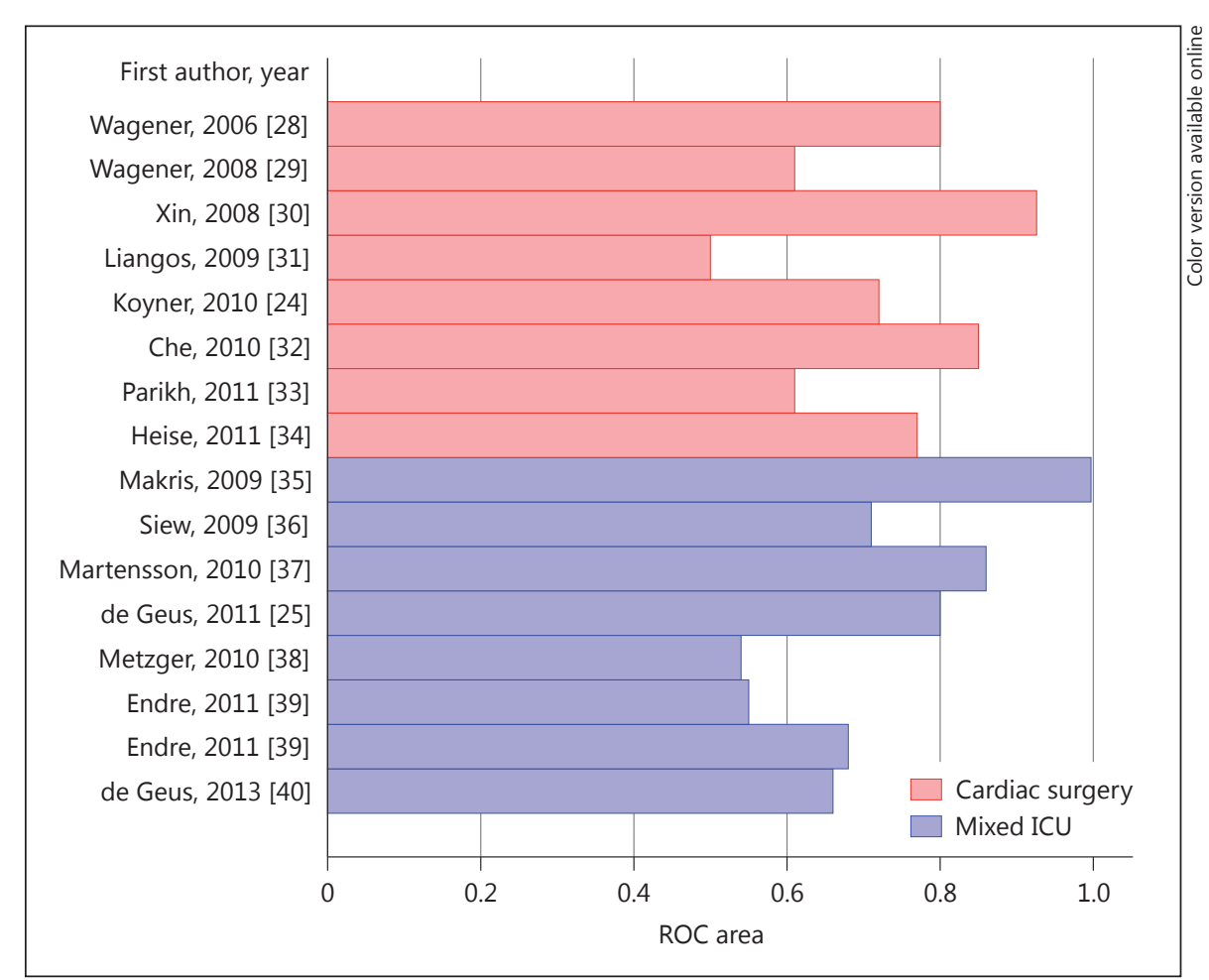

ranging from 0.50 to 0.93 after cardiac surgery [24, 2834 ] and from 0.54 to 0.99 in general ICU patients [25, 35-40] (fig. 1). Although the performance increases with AKI severity [25], the ROC areas for predicting the progression to severe AKI (RIFLE I and/or AKIN stage 2) was $<0.70$ in the most recent studies $[26,41]$.

The non-consistent performance across studies in critically ill adult patients can be explained by several factors. First, comparing a biomarker of assumed parenchymal kidney injury against a reference method (i.e. creatinine) that imperfectly estimates functional kidney impairment (clinical AKI) is problematic. Due to continuing loss of muscle mass in the critically ill [42] and dilution of serum creatinine in fluid-loaded patients [43], AKI might go undetected by conventional creatinine-based criteria. Emerging evidence shows that patients with elevated NGAL in the absence of creatinine-based criteria for AKI carry an increased risk of adverse events including need for renal replacement therapy and death $[44,45]$. Whether this specifically represents subclinical AKI or is simply an expression of severe systemicinflammation is yet to be determined.

Second, the burden of comorbidities, especially chronic kidney disease (CKD), may affect the results since CKD per se is associated with elevated serum and urinary NGAL levels [46, 47]. In fact, McIlroy et al. [48] found that NGAL only predicted AKI in patients with normal baseline renal function. The notion that comorbidities are important confounders in the studies is further supported by the fact that the predictive performance is generally better in children [49].

Third, the time from biomarker measurement to AKI diagnosis differs substantially among studies. It could be expected that predictive values would increase when NGAL is measured closer to the time of insult. This relation is not, however, seen in the studies. Notably, Kashani et al. [26] found only a limited performance (ROC area 0.69 ) when NGAL was used to predict severe AKI within $12 \mathrm{~h}$ in a large study.

Finally, existing studies are affected by the limited ability of the NGAL assays to distinguish between the various molecular forms released by different tissues. Systemic inflammation triggered by conditions such as sepsis or procedures like cardiopulmonary bypass (CPB) is strongly associated with AKI development [50]. At the same time, such inflammatory triggers will activate circulating neutrophils to release their granular contents, including NGAL. In addition, NGAL synthesis increases in nonhaematopoietic cells in various tissues such as the lung [51] and the liver [18] as part of the inflammatory response. The resultant increase in plasma NGAL will in- 
Fig. 2. Nature and source of NGAL in plasma and urine during AKI. Systemic inflammation induces NGAL synthesis by extrarenal tissues and the release of NGAL from neutrophils. Urinary NGAL increases due to an impaired reabsorption of the filtered load (downregulation of megalin-cubulin receptors in the proximal tubule), increased synthesis from stressed tubular cells in the distal nephron and release by infiltrating neutrophils. Neutrophils mainly release the dimeric form (and to some extent the monomeric form), whereas tubular cells mainly produce the monomeric form and to some extent NGAL conjugated with MMP-9 (heterodimeric NGAL).

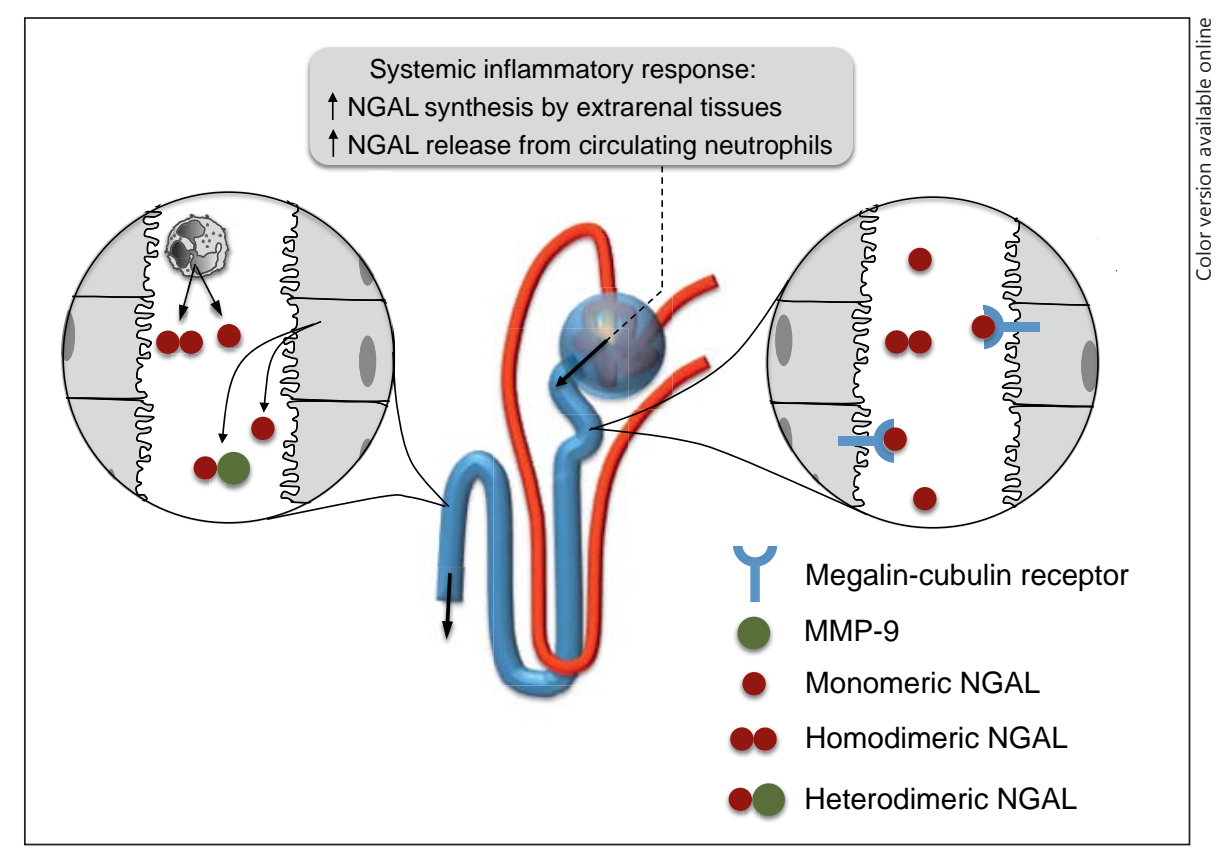

crease the filtered load and hence urinary levels irrespective of any potential kidney damage. This is supported by studies showing elevated levels in plasma [52] and urine [53] after cardiac surgery in patients without postoperative AKI. The fact that NGAL levels are correlated with $\mathrm{CPB}$ time and that homodimeric NGAL is the predominant form in these patients' urine [53] suggests that the neutrophils, rather than the kidney tubular cells, are the main source. Similar conclusions can be drawn from general ICU populations where sepsis is associated with elevated plasma NGAL independent of degree of renal impairment [37, 54].

Based on these findings, NGAL might be a more specific AKI marker in patient populations where the 'noise' from severe systemic inflammation and multi-organ damage is less pronounced. In fact, this was shown in a 635-patient emergency department cohort where only $6 \%$ were admitted to the ICU [55]. The authors found that urinary NGAL distinguished sustained AKI (RIFLE R for at least 3 days despite fluid resuscitation) from non-AKI with or without concomitant stable CKD with high sensitivity (90.0\%) and specificity (99.5\%). The prevalence and hence the possible confounding effect of sepsis was low in this study, which likely contributed to the excellent predictive values. A similar study investigating septic patients on emergency department admission found plasma NGAL to be a sensitive (sensitivity 0.96 ) but non-specific (specificity 0.51) AKI predictor [56].
Under normal conditions, filtered NGAL is almost completely reabsorbed by the proximal tubules via megalin-cubulin receptor-mediated endocytosis, resulting in minimal urinary NGAL levels $[21,57]$. The total concentration of urinary NGAL in AKI probably represents a mixture of different molecular forms of NGAL with different cellular origins (fig. 2). Monomeric NGAL increase either due to an induced synthesis in the tubular cells or as an effect of impaired reabsorption of the filtered load produced by extrarenal tissues [21, 58]. Furthermore, infiltration of neutrophils in the kidney has been observed in both animal models and in biopsy specimens from patients with AKI $[59,60]$. Dimeric NGAL in urine might emanate from these infiltrating neutrophils, but glomerular filtration of dimeric NGAL released from activated neutrophils in the circulation, e.g. in septic patients, may also contribute.

The different forms of NGAL (monomeric, dimeric, heterodimeric) expose different epitopes and the configuration of antibodies will have an impact on the clinical performance of the assay [53]. Notably, most studies use commercial NGAL assays such as the BioPorto (Gentofte, Denmark) and R\&D Systems (Minneapolis, Minn., USA) assays. Unfortunately, these assays are unable to specifically measure NGAL secreted by stressed or injured tubular cells. Although a dimer-specific assay with the potential to quantify neutrophil-derived NGAL was recently proposed, efforts to amplify the monomeric, tubular cell-specific signal have been less successful $[15,61]$. 


\section{The Fall of NGAL as a Kidney-Specific Injury Marker in Critical Illness}

The fervent introduction of presumed tissue-specific biomarkers has pervaded the field of intensive care medicine for many years. These biomarkers have traditionally been discovered and validated in patients with an isolated organ injury. Indeed, biomarkers of acute ischaemic myocardial injury (troponins), heart failure (brain natriuretic peptide), traumatic brain injury (S-100) and ischaemic brain injury (neuron-specific enolase) are of added value in the decision process and their use may even affect outcomes in selected patient populations [62-65]. However, ICU patients at risk of AKI suffer from a strong inflammatory response with stress/injuries in several organs, are exposed to repeated invasive procedures, fluid therapy and vasopressor support, blood transfusion and multiple other biological modifiers.

Therefore, it is not surprising that tissue-specific biomarker signals drown in the sea of such confounders. Indeed, 'biomarkeraemia' is frequently seen in ICU patients and is likely a signal of overall illness severity rather than specific organ damage [65-67].

Now, NGAL can be added to the existing list of biomarkers of single organ injury that appear to perform well in specific populations but show a major loss of diagnostic accuracy when used in the ICU, where they likely represent yet another biological expression of illness severity.

\section{Conclusions}

Since the rise of NGAL as a potential biomarker of early AKI, beginning for more than a decade ago, numerous studies have investigated its role in various clinical situations. However, the idea of NGAL as a troponin for the kidney has fallen as the understanding of the molecule's complex nature has emerged. NGAL levels increase unpredictably during evolving AKI but also during other chronic and acute inflammatory conditions frequently encountered in the ICU, such as in sepsis, after CPB surgery, in CKDs and during urinary tract infections and acute exacerbations of obstructive pulmonary diseases. Therefore, adding NGAL to the clinical evaluation of critically ill patients with or at risk of AKI has to wait until assays measuring kidney-specific NGAL are available. Simultaneously, the ability of dimeric, neutrophil-derived NGAL to detect severe sepsis, the most common trigger of AKI in the ICU, needs to be further explored. Until such studies are completed, NGAL measurement, which had shown such promise early on, should be confined to research protocols and to the assessment of selected emergency department patients.

\section{References}

1 Mishra J, Ma Q, Prada A, et al: Identification of neutrophil gelatinase-associated lipocalin as a novel early urinary biomarker for ischemic renal injury. J Am Soc Nephrol 2003;14: 2534-2543.

-2 Mishra J, Mori K, Ma Q, Kelly C, Barasch J, Devarajan P: Neutrophil gelatinase-associated lipocalin: a novel early urinary biomarker for cisplatin nephrotoxicity. Am J Nephrol 2004;24:307-315.

-3 Mishra J, Dent C, Tarabishi R, et al: Neutrophil gelatinase-associated lipocalin as a biomarker for acute renal injury after cardiac surgery. Lancet 2005;365:1231-1238.

4 Devarajan P: Review: neutrophil gelatinaseassociated lipocalin: a troponin-like biomarker for human acute kidney injury. Nephrology (Carlton) 2010;15:419-428.

5 Akcay A, Nguyen Q, Edelstein CL: Mediators of inflammation in acute kidney injury. Mediators Inflamm 2009;2009:137072.

6 Cowland JB, Borregaard N: Molecular characterization and pattern of tissue expression of the gene for neutrophil gelatinase-associated lipocalin from humans. Genomics 1997; 45:17-23.

7 Cai L, Rubin J, Han W, Venge P, Xu S: The origin of multiple molecular forms in urine of HNL/NGAL. Clin J Am Soc Nephrol 2010;5: 2229-2235.

-8 Kjeldsen L, Johnsen AH, Sengelov H, Borregaard $\mathrm{N}$ : Isolation and primary structure of NGAL, a novel protein associated with human neutrophil gelatinase. J Biol Chem 1993; 268:10425-10432.

-9 Xu SY, Carlson M, Engstrom A, Garcia R, Peterson CG, Venge P: Purification and characterization of a human neutrophil lipocalin from the secondary granules of human neutrophils. Scand J Clin Lab Invest 1994;54:365376.

10 Borregaard N, Cowland JB: Granules of the human neutrophilic polymorphonuclear leukocyte. Blood 1997;89:3503-3521.

11 Cowland JB, Sorensen OE, Sehested M, Borregaard N: Neutrophil gelatinase-associated lipocalin is upregulated in human epithelial cells by IL- $1 \beta$, but not by TNF- $\alpha$. J Immunol 2003;171:6630-6639.

$12 \mathrm{Xu} \mathrm{S}$, Venge P: Lipocalins as biochemical markers of disease. Biochim Biophys Acta 2000;1482:298-307.

13 Xu SY, Pauksen K, Venge P: Serum measurements of human neutrophil lipocalin discriminate between acute bacterial and viral infections. Scand J Clin Lab Invest 1995;55:125131.

14 Fjaertoft G, Foucard T, Xu S, Venge P: Human neutrophil lipocalin as a diagnostic tool in children with acute infections: a study of the kinetics. Acta Paediatr 2005;94:661-666.

-15 Martensson J, Xu S, Bell M, Martling CR, Venge P: Immunoassays distinguishing between HNL/NGAL released in urine from kidney epithelial cells and neutrophils. Clin Chim Acta 2012;413:1661-1667.

16 Schmidt-Ott KM, Mori K, Li JY, et al: Dual action of neutrophil gelatinase-associated lipocalin. J Am Soc Nephrol 2007;18:407-413. 
17 Goetz DH, Holmes MA, Borregaard N, Bluhm ME, Raymond KN, Strong RK: The neutrophil lipocalin NGAL is a bacteriostatic agent that interferes with siderophore-mediated iron acquisition. Mol Cell 2002;10:10331043.

18 Flo TH, Smith KD, Sato S, et al: Lipocalin 2 mediates an innate immune response to bacterial infection by sequestrating iron. Nature 2004;432:917-921.

19 Yang J, Goetz D, Li JY, et al: An iron delivery pathway mediated by a lipocalin. Mol Cell 2002;10:1045-1056.

20 Mishra J, Mori K, Ma Q, et al: Amelioration of ischemic acute renal injury by neutrophil gelatinase-associated lipocalin. J Am Soc Nephrol 2004; 15:3073-3082.

21 Mori K, Lee HT, Rapoport D, et al: Endocytic delivery of lipocalin-siderophore-iron complex rescues the kidney from ischemia-reperfusion injury. J Clin Invest 2005;115:610-621.

-22 Supavekin S, Zhang W, Kucherlapati R, Kaskel FJ, Moore LC, Devarajan P: Differential gene expression following early renal ischemia/reperfusion. Kidney Int 2003;63:1714-1724.

-23 Zappitelli M, Washburn KK, Arikan AA, et al: Urine neutrophil gelatinase-associated lipocalin is an early marker of acute kidney injury in critically ill children: a prospective cohort study. Crit Care 2007;11:R84.

-24 Koyner JL, Vaidya VS, Bennett MR, et al: Urinary biomarkers in the clinical prognosis and early detection of acute kidney injury. Clin J Am Soc Nephrol 2010;5:2154-2165.

-25 de Geus HR, Bakker J, Lesaffre EM, Le Noble JL: Neutrophil gelatinase-associated lipocalin at ICU admission predicts for acute kidney injury in adult patients. Am J Respir Crit Care Med 2011;183:907-914.

26 Kashani K, Al-Khafaji A, Ardiles T, et al: Discovery and validation of cell cycle arrest biomarkers in human acute kidney injury. Crit Care 2013;17:R25.

27 Haase M, Bellomo R, Devarajan P, et al: Novel biomarkers early predict the severity of acute kidney injury after cardiac surgery in adults. Ann Thorac Surg 2009;88:124-130.

-28 Wagener G, Jan M, Kim M, et al: Association between increases in urinary neutrophil gelatinase-associated lipocalin and acute renal dysfunction after adult cardiac surgery. Anesthesiology 2006;105:485-491.

-29 Wagener G, Gubitosa G, Wang S, Borregaard N, Kim M, Lee HT: Urinary neutrophil gelatinase-associated lipocalin and acute kidney injury after cardiac surgery. Am J Kidney Dis 2008;52:425-433.

- 30 Xin C, Yulong X, Yu C, Changchun C, Feng $\mathrm{Z}$, Xinwei M: Urine neutrophil gelatinase-associated lipocalin and interleukin-18 predict acute kidney injury after cardiac surgery. Ren Fail 2008;30:904-913.

- 31 Liangos O, Tighiouart H, Perianayagam MC, et al: Comparative analysis of urinary biomarkers for early detection of acute kidney injury following cardiopulmonary bypass. Biomarkers 2009; 14:423-431.
2 Che M, Xie B, Xue S, et al: Clinical usefulness of novel biomarkers for the detection of acute kidney injury following elective cardiac surgery. Nephron Clin Pract 2010;115:c66-c72.

33 Parikh CR, Coca SG, Thiessen-Philbrook H, et al: Postoperative biomarkers predict acute kidney injury and poor outcomes after adult cardiac surgery. J Am Soc Nephrol 2011;22: 1748-1757.

- 34 Heise D, Rentsch K, Braeuer A, Friedrich M, Quintel M: Comparison of urinary neutrophil glucosaminidase-associated lipocalin, cystatin C, and $\alpha_{1}$-microglobulin for early detection of acute renal injury after cardiac surgery. Eur J Cardiothorac Surg 2011;39:38-43.

35 Makris K, Markou N, Evodia E, et al: Urinary neutrophil gelatinase-associated lipocalin as an early marker of acute kidney injury in critically ill multiple trauma patients. Clin Chem Lab Med 2009;47:79-82.

-36 Siew ED, Ware LB, Gebretsadik T, et al: Urine neutrophil gelatinase-associated lipocalin moderately predicts acute kidney injury in critically ill adults. J Am Soc Nephrol 2009;20: 1823-1832.

37 Martensson J, Bell M, Oldner A, Xu S, Venge $P$, Martling CR: Neutrophil gelatinase-associated lipocalin in adult septic patients with and without acute kidney injury. Intensive Care Med 2010;36:1333-1340.

38 Metzger J, Kirsch T, Schiffer E, et al: Urinary excretion of twenty peptides forms an early and accurate diagnostic pattern of acute kidney injury. Kidney Int 2010;78:1252-1262.

39 Endre ZH, Pickering JW, Walker RJ, et al: Improved performance of urinary biomarkers of acute kidney injury in the critically ill by stratification for injury duration and baseline renal function. Kidney Int 2011;79;11191130.

40 de Geus HR, Fortrie G, Betjes MG, Van Schaik $\mathrm{RH}$, Groeneveld AB: Time of injury affects urinary biomarker predictive values for acute kidney injury in critically ill, non-septic patients. BMC Nephrol 2013;14:273.

- 41 Koyner JL, Garg AX, Coca SG, et al: Biomarkers predict progression of acute kidney injury after cardiac surgery. J Am Soc Nephrol 2012; 23:905-914.

42 Puthucheary ZA, Rawal J, McPhail M, et al: Acute skeletal muscle wasting in critical illness. JAMA 2013;310:1591-1600.

43 Liu KD, Thompson BT, Ancukiewicz M, et al: Acute kidney injury in patients with acute lung injury: impact of fluid accumulation on classification of acute kidney injury and associated outcomes. Crit Care Med 2011;39: 2665-2671.

44 Haase M, Devarajan P, Haase-Fielitz A, et al: The outcome of neutrophil gelatinase-associated lipocalin-positive subclinical acute kidney injury: a multicenter pooled analysis of prospective studies. J Am Coll Cardiol 2011; 57:1752-1761.

45 Nickolas TL, Schmidt-Ott KM, Canetta P, et al: Diagnostic and prognostic stratification in the emergency department using urinary bio- markers of nephron damage: a multicenter prospective cohort study. J Am Coll Cardiol 2012;59:246-255.

-46 Mitsnefes MM, Kathman TS, Mishra J, et al: Serum neutrophil gelatinase-associated lipocalin as a marker of renal function in children with chronic kidney disease. Pediatr Nephrol 2007;22:101-108.

-47 Bolignano D, Lacquaniti A, Coppolino G, et al: Neutrophil gelatinase-associated lipocalin and progression of chronic kidney disease. Clin J Am Soc Nephrol 2009;4:337-344.

48 McIlroy DR, Wagener G, Lee HT: Neutrophil gelatinase-associated lipocalin and acute kidney injury after cardiac surgery: the effect of baseline renal function on diagnostic performance. Clin J Am Soc Nephrol 2010;5:211219.

49 Haase M, Bellomo R, Devarajan P, Schlattmann P, Haase-Fielitz A: Accuracy of neutrophil gelatinase-associated lipocalin in diagnosis and prognosis in acute kidney injury: a systematic review and meta-analysis. Am J Kidney Dis 2009;54:1012-1024.

50 Uchino S, Kellum JA, Bellomo R, et al: Acute renal failure in critically ill patients: a multinational, multicenter study. JAMA 2005;294: 813-818.

51 Grigoryev DN, Liu M, Hassoun HT, Cheadle C, Barnes KC, Rabb H: The local and systemic inflammatory transcriptome after acute kidney injury. J Am Soc Nephrol 2008; 19: 547-558.

52 Koyner JL, Bennett MR, Worcester EM, et al: Urinary cystatin $\mathrm{C}$ as an early biomarker of acute kidney injury following adult cardiothoracic surgery. Kidney Int 2008;74:10591069.

53 Cai L, Borowiec J, Xu S, Han W, Venge P: Assays of urine levels of HNL/NGAL in patients undergoing cardiac surgery and the impact of antibody configuration on their clinical performances. Clin Chim Acta 2009;403:121125 .

54 Martensson J, Bell M, Xu S, et al: Association of plasma neutrophil gelatinase-associated lipocalin with sepsis and acute kidney dysfunction. Biomarkers 2013;18:349-356.

55 Nickolas TL, O’Rourke MJ, Yang J, et al: Sensitivity and specificity of a single emergency department measurement of urinary neutrophil gelatinase-associated lipocalin for diagnosing acute kidney injury. Ann Intern Med 2008;148:810-819.

56 Shapiro NI, Trzeciak S, Hollander JE, et al: The diagnostic accuracy of plasma neutrophil gelatinase-associated lipocalin in the prediction of acute kidney injury in emergency department patients with suspected sepsis. Ann Emerg Med 2010;56:52-59 e51.

57 Hvidberg V, Jacobsen C, Strong RK, Cowland JB, Moestrup SK, Borregaard N: The endocytic receptor megalin binds the iron transporting neutrophil-gelatinase-associated lipocalin with high affinity and mediates its cellular uptake. FEBS Lett 2005;579:773777. 
58 Kuwabara T, Mori K, Mukoyama M, et al: Urinary neutrophil gelatinase-associated lipocalin levels reflect damage to glomeruli, proximal tubules, and distal nephrons. Kidney Int 2009;75:285-294.

59 Awad AS, Rouse M, Huang L, et al: Compartmentalization of neutrophils in the kidney and lung following acute ischemic kidney injury. Kidney Int 2009;75:689-698.

60 Lerolle N, Nochy D, Guerot E, et al: Histopathology of septic shock induced acute kidney injury: apoptosis and leukocytic infiltration. Intensive Care Med 2010;36:471-478.
61 Glassford NJ, Schneider AG, Xu S, et al: The nature and discriminatory value of urinary neutrophil gelatinase-associated lipocalin in critically ill patients at risk of acute kidney injury. Intensive Care Med 2013;39:1714-1724.

62 Åberg A: Myocardial Infarctions in Sweden 1987-2005. Stockholm, Official Statistics of Sweden, 2008, pp 1-102.

63 Mahadavan G, Nguyen TH, Horowitz JD: Brain natriuretic peptide: a biomarker for all cardiac disease? Curr Opin Cardiol 2014;29: 160-166.
64 Mercier E, Boutin A, Lauzier F, et al: Predictive value of $S-100 \beta$ protein for prognosis in patients with moderate and severe traumatic brain injury: systematic review and metaanalysis. BMJ 2013;346:f1757.

65 Taccone FS, Cronberg T, Friberg H, et al: How to assess prognosis after cardiac arrest and therapeutic hypothermia. Crit Care 2014; 18:202.

66 Markou N, Gregorakos L, Myrianthefs P: Increased blood troponin levels in ICU patients. Curr Opin Crit Care 2011;17:454-463.

-67 Noveanu M, Mebazaa A, Mueller C: Cardiovascular biomarkers in the ICU. Curr Opin Crit Care 2009;15:377-383. 